\title{
Entrenamiento propioceptivo para futbolistas, una propuesta desde la fisioterapia.
}

\author{
Proprioceptive training for soccer players: a physical therapy proposal
}

Manuel Alberto Riveros Medina**

\section{Resumen}

Este artículo muestra la importancia de la reeducación propioceptiva en el futbolista como factor fundamental para la prevención de lesiones, la pronta recuperación y la disminución de los niveles de reincidencia de la misma, además de contribuir al mejoramiento de la capacidad cinética del individuo de acuerdo con las demandas específicas del deporte.

Tambien se muestra cómo el fisioterapeuta se convierte en motor principal para la planificación y el control de programas de reeducación propioceptiva de acuerdo con las necesidades individuales del futbolista, desarrollándolas progresivamente a través de etapas especificas.

Palabras clave: Deporte, fútbol, propiocepción, entrenamiento, reeducación, fisioterapeuta.

\begin{abstract}
This article shows the importance of proprioceptive re-education in soccer players as a basic factor to prevent lesions, promote soon recovery, and reduce recurrent lesions. It also points out the contribution of proprioceptive re-education to the improvement of an individual's kinetic capability according to the specific demands of soccer.

The article also explains how the physical therapist becomes the most important driving force in proprioceptive reeducation programs planning and management, in accordance with a player's individual needs, developing them through successive specific stages.
\end{abstract}

Key words: $\quad$ Sport, soccer, proprioception, training, reeducation, physical therapist.

Fecha de recepción: 26 de octubre de 2006

Fecha de aceptación: 28 de noviembre de 2006

1 Ponencia realizada en el I Congreso Internacional de de Fisioterapia. San José de Costa Rica. 2002.

** Licenciado en Educación Física de la Universidad Pedagógica Nacional. Fisioterapeuta de la Escuela Colombiana de Rehabilitación. Especialista en el Ejercicio Físico para la Salud de la Universidad del Rosario. Fisioterapeuta con énfasis en deporte Docente e investigador universitario en el área de Biomecánica. Actualmente es director del proyecto de investigación Análisis Biomecánico 3D para la estandarización de la técnica deportiva desde la óptica fisioterapéutica en diferentes disciplinas en deportistas de rendimiento, en el Programa de Fisioterapia de la Universidad Manuela Beltrán, UMB.

Docente en la Facultad de Educación Física de la Universidad Pedagógica Nacional.

Docente de Biomecánica y Anatomía Funcional en la Facultad de Cultura Física de la Universidad Santo Tomás de Aquino. rivermedfis@gmail.com 
El deporte evoluciona constantemente, lo cual exige el mejoramiento continuo del rendimiento, donde se estimulan no sólo factores de tipo social, sino también psicológico, anatómico y funcional.

En el fútbol, caso específico del presente artículo, hace referencia a que "los nuevos sistemas de juego y el actual modo de entenderlo, a partir de las modificaciones técnicas y debido a la constante introducción del deporte en las ciencias biológicas, requieren unas capacidades de rendimiento elevadas" (Bosco), además de la interacción de los diferentes sistemas corporales (músculo-esquelético, nervioso, cardiaco y pulmonar) que conducen al logro del objetivo, reflejado en la competencia.

En los deportes de conjunto, como en el fútbol,

la habilidad técnica y táctica del jugador es un factor indispensable para una realización concreta y real del juego mismo. La habilidad motriz y la inteligencia neuromuscular, mediante la regulación plástica del sistema nervioso, se concretan y se realizan prácticamente gracias a adaptaciones de naturaleza cognitiva y perceptiva que se manifiestan en el terreno de juego bajo formas de respuestas espacio-temporales y modificaciones cinéticas (Bosco).

Por tanto, el fútbol como deporte requiere para su desarrollo total una serie de elementos tanto fisiológicos como cinéticos, donde deben ubicarse las cualidades físicas (fuerza, resistencia, velocidad, flexibilidad; coordinación, equilibrio, velocidad de desplazamiento y reacción, movilidad), al igual que los patrones básicos de movimiento, PBM, como caminar correr, saltar, patear, lanzar y combinaciones de éstos. A ellos también se suman otras actividades motrices típicas de este deporte, como son: desplazamientos en diferentes direcciones, desplazamientos con aceleraciones desde quietud o movimiento y desaceleraciones; cambios de dirección (acciones repentinas de movimiento) con sus virajes y fintas, ejecutadas con diferentes ritmos y mediadas ya sea por la voluntad propia o de acuerdo con las circunstancias momentáneas y cambiantes del juego (por ejemplo: factores externos en los cuales se destaca el compañero, el adversario, o, en el caso del salto, la brevedad del tiempo de preparación de éste, la no elección del momento del salto, el no estar en la posición adecuada para la ejecución, entre otras).

Unido a esto, la necesidad del futbolista de ejecutar las tareas de movimiento específicas de su deporte dentro de una gran exigencia donde las características cualitativas del movimiento (precisión, amplitud, fluidez y ritmo) son expuestas en un marco de juego intenso, dinámico, cambiante y de gran diversidad, que lo convierten en una práctica compleja que requiere el desarrollo máximo de sus cualidades físicas sincronizadas con habilidades y destrezas específicas propias de dicho deporte.

Para que dichas acciones fisiológicas y cinéticas se den de manera adecuada, debe existir una interacción entre el sistema de palancas que conforma el cuerpo humano y en el cual los músculos, los huesos y las articulaciones actúan conjuntamente con un regulador como es el sistema nervioso, facilitando la transmisión de impulsos aferentes y eferentes, regulando y estableciendo una armonía en el control del movimiento. Aqui también desempeña un papel fundamental el sistema propioceptivo del futbolista, ya que constituye una de las bases aferentes más importantes para la creación de un engrama de movimiento efectivo y ajustado a los requerimientos del fútbol; de lo contrario, la deficiencia o alteración de este sistema podrá ser un factor de riesgo importante de lesión osteomuscular.

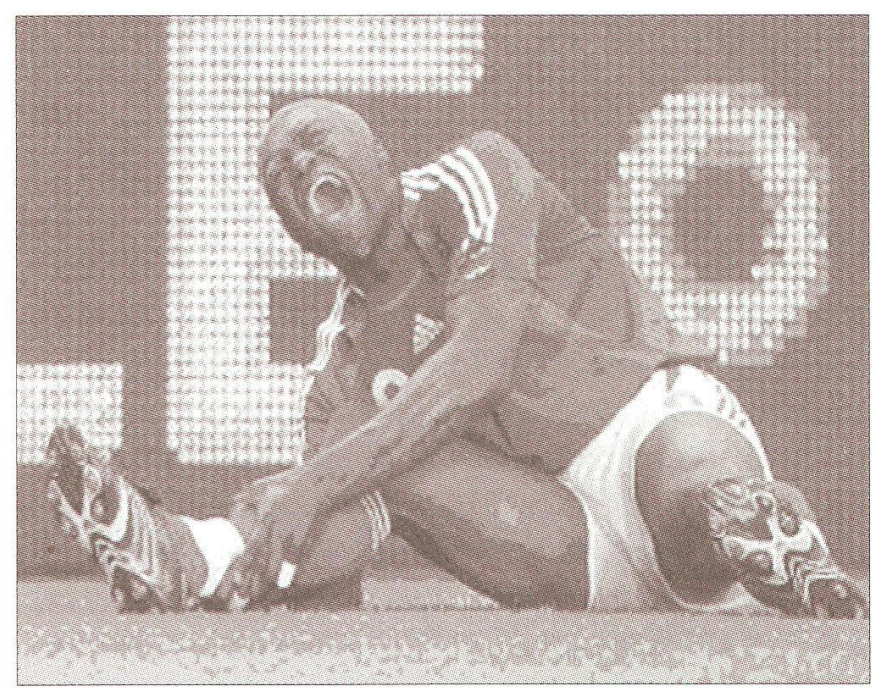

Tomado de: www.fifa.com (2006).

Al ser la propiocepción una función fundamental del sistema nervioso, se hace necesario hacer referencia al mismo. Veamos:

\section{El sistema nervioso}

El sistema nervioso es el centro de regulación y la red de comunicaciones del cuerpo, y cumple con tres funciones generales:

1. Sensorial, con la cual detecta los cambios del propio cuerpo y del medio externo 
2. Integradora, con la cual interpreta la información captada.

3. Motriz, con la cual responde a la interpretación mediante acciones consistentes en contracciones musculares o secreciones glandulares.

Gracias a estas tres funciones el sistema nervioso es el medio más rápido de que dispone el organismo para el mantenimiento de la homeostasis.

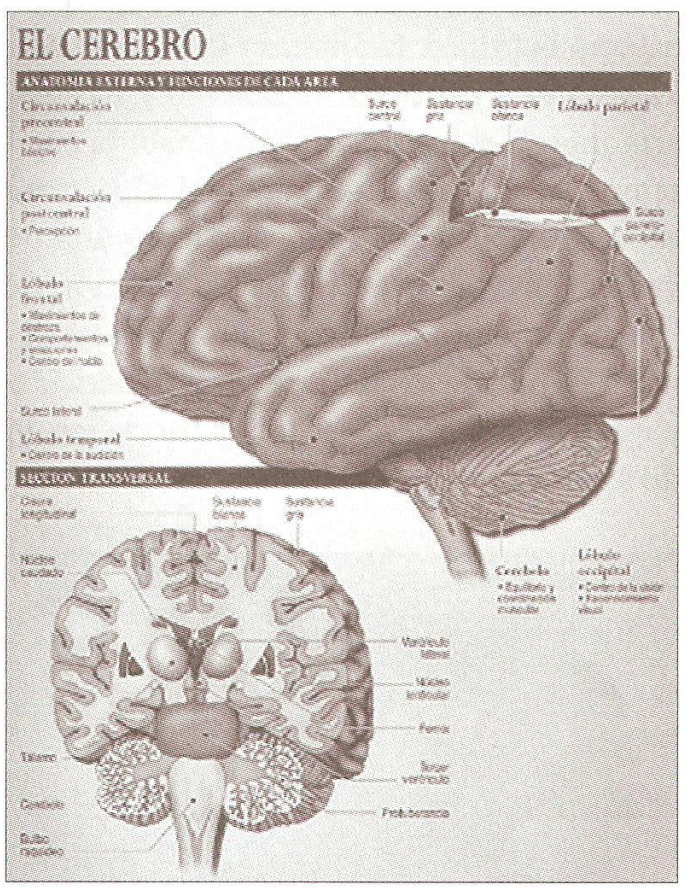

En el ser humano las modificaciones del medio ambiente o del propio organismo son fuente de múltiples y diversos estímulos que actúan sobre órganos receptores que, de acuerdo con la naturaleza del estímulo, llegan a centros que permiten su conocimiento y su discriminación. La sensación, es decir, el fenómeno producido por

la acción de un estímulo sobre un órgano receptor es el que le permite al organismo conocer.

Las sensaciones pueden dividirse en:

1. Superficiales: tacto, dolor, temperatura.

2. Profundas: que corresponden a la posición muscular y articular (propiocepción), al dolor muscular profundo y a la vibración.

3. Corticales: correspondientes a la esterognosia, grafestesia, barognosia, etcétera.

Además, existen receptores sensoriales, esto es, células especializadas que registran cambios particulares en el medio ambiente, y se clasifican en:

1. Exteroceptores: responden a cambios sucedidos en el medio ambiente.
2. Interoceptores: responden a cambios en los tejidos viscerales, de los vasos sanguíneos y cambios químicos.

3. Propioceptores: regulan el tono postural y la acción muscular.

\section{Definición y fundamentos de la propiocepción}

La propiocepción es un tipo de sensación profunda que se da a partir de la recepción de estímulos dados por los receptores musculares, tendinosos y articulares denominados propioceptores (huso neuromuscular, órgano tendinoso de Golgi, receptores articulares, tipo I, II, III, IV), que están enmarcados en la función sensorial del sistema nervioso.

La propiocepción corresponde al sentido cinestési$\mathrm{co}$, es decir, confiere al aparato neuromuscular retroalimentación de la rapidez, la dirección y la dinámica del movimiento, así como del sentido de la posición estática que significa la orientación consciente de las distintas partes del cuerpo con relación a las otras.

Mount Castle y Willis (1980) definen la propiocepción como la conciencia de la posición de las extremidades, y la cinestesia como la conciencia del movimiento de las articulaciones.

Sherrington (1906) describe la propiocepción como un sentido que incluye sensaciones vestibulares e información de los músculos y las articulaciones que no tienen que ser percibidas necesariamente.

Scott Lephart (1998) la define como una variación especializada de la modalidad sensorial del tacto, que abarca las sensaciones de movimiento (cinestesia) y la posición de las articulaciones (sentido de posición de las articulaciones).

Por tanto, la propiocepción es la habilidad del cuerpo para responder a un estímulo externo a través de ajustes musculares reflejos, observados en respuestas espaciotemporales y modificaciones cinéticas, mediados por mecano-receptores (receptores articulares y musculares) que contribuyen al ajuste del control neuromuscular $y$, por ende, a la generación de engramas de movimiento efectivos y ajustados a las necesidades del individuo, ya sea para el funcionamiento articular o para la modulación de la función muscular.

La propiocepción es fundamental para el futbolista, pues la exigencia motriz y física de su accionar en el campo, más la diversidad de estímulos generados por las circunstancias momentáneas y cambiantes del juego, llevan a que éste sea un factor básico para las grandes demandas cinéticas del fútbol, que facilita la tarea mo- 
triz eficiente. Por el contrario, su alteración o su deficiencia, pueden promover lesiones del deportista.

En el fútbol los miembros inferiores son el eje principal de la actividad; desempeñan un papel fundamental en la técnica deportiva, a la vez que cumplen con dos funciones importantes en la homocinética: el soporte de peso corporal y la locomoción. Para que estas funciones se den adecuadamente, se requiere cumplir con todas las cualidades anatomo-funcionales y biomecánicas del pie y de los miembros inferiores, además de una buena integración del sistema propioceptivo como receptor de la información externa de posición y movimiento que le permite dar respuestas cinéticas de adaptación a los estímulos externos. Por ejemplo, en el caso específico del pie, éste cumple con su triple cometido:

1. Como órgano de soporte del cuerpo en la marcha, la carrera y el salto.

2. Como palanca que permite elevar e impulsar el cuerpo (acción propulsora).

3. Como amortiguador y equilibrador de las fuerzas que actúan sobre sí mismos (Zintl, 1991).

De acuerdo con lo expuesto, es importante que el futbolista realice un entrenamiento propioceptivo dirigido por el fisioterapeuta, de manera que contribuya a dar respuesta adecuada a las grandes demandas cinéticas a que se ven sometidos sus miembros inferiores, y a cumplir con los dos objetivos principales de la rehabilitación deportiva: la prevención de lesiones y el retorno pronto y seguro del deportista a su actividad, en condiciones iguales o mejores que las presentadas antes de la lesión.

Para generar el programa de entrenamiento propioceptivo para futbolistas, debe hacerse énfasis en sus miembros inferiores, teniendo también en cuenta la in-

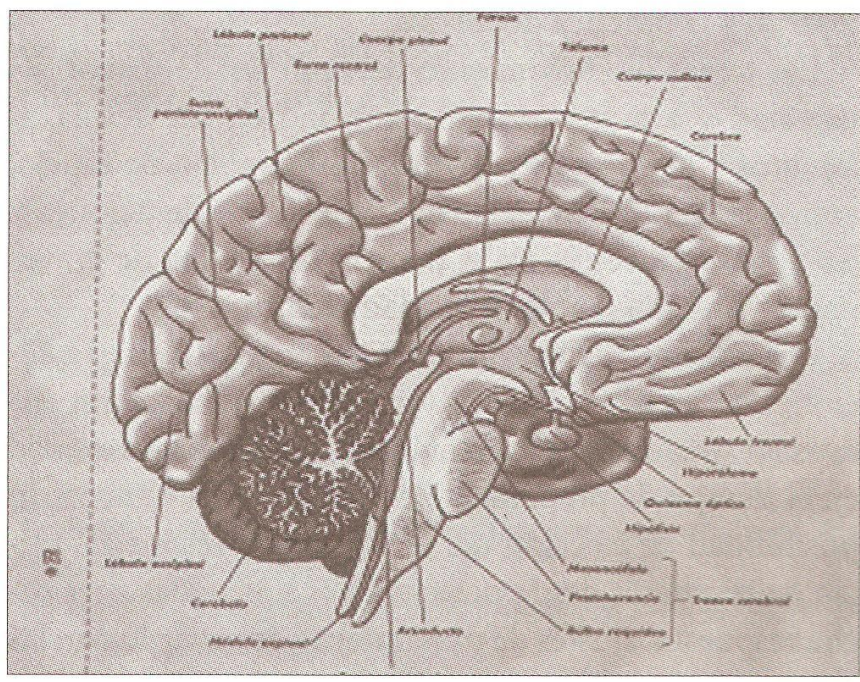

fluencia del sistema nervioso central en las actividades motrices a través de tres niveles de control motor: médula espinal, tallo encefálico y sistema nervioso central, donde se incluyen la corteza motora, los ganglios basales y el cerebelo.

En el primer nivel, ubicado en la médula espinal, se emiten patrones de movimiento a partir de los reflejos que contribuyen a generar la fijación refleja (ajuste muscular reflejo), durante condiciones de tensión superior a la que habitualmente se presenta en la articulación. Además, los husos neuromusculares cumplen una función importante en el control del movimiento muscular ajustando la actividad de las motoneuronas inferiores. Por consiguiente, en este nivel las actividades por realizar deben propiciar la estabilización refleja o dinámica de la articulación, es decir actividades súbitas o imprevistas de la posición articular.

El segundo nivel de control motor está ubicado en el tallo encefálico, donde se busca que los propioceptores articulares y los centros vestibulares en los oídos (utrículo, sáculo y canales semicirculares superior, externo y posterior) y de los ojos (conos y bastoncillos) mantengan la postura y el equilibrio corporales. Las actividades por desarrollar para estimular este nivel corresponden a ejecuciones de postura y equilibrio con información visual o sin ella.

El tercer nivel, el más elevado de la función del sistema nervioso central, está mediado por la conciencia cognoscitiva de la posición y el movimiento corporales. A este nivel se inician y se programan engramas motores para movimientos de carácter voluntario y consciente, que con la estimulación se convertirán en inconscientes en el programa motor (destreza) (Kandel y Schwartzs, 1991; Downie, 1993).

Por tanto, lo que se busca fisiológicamente con el programa de entrenamiento propioceptivo para futbolistas es entrenar las vías aferentes, alteradas o no, y así lograr un aumento de la sensación de movimiento y posición articular en los miembros inferiores, a través de actividades o tareas de movimiento que serán programadas o planificadas sobre la base de los principios del entrenamiento deportivo (Guyton, 1991).

\section{Actividades o tareas de movimiento}

\section{Etapa 1}

Se proponen tareas de restablecimiento de la estabilización dinámica de la articulación, el equilibrio y la conciencia propioceptiva a través de actividades de soporte y transferencia de peso, sobre diversas superficies esta- 
bles (blandas y duras entre las cuales se pueden encontrar arena, grama, etc.). Posteriormente se debe acceder a superficies inestables; se realiza ascenso y descenso de rampas y escaleras hacia delante y hacia atrás; se realiza soporte bipedal evolucionando hacia monopedal con ojos abiertos y después con ojos cerrados.

También se ejecutan actividades de marcha donde los desplazamientos se harán anteriores, posteriores, laterales y diagonales; luego se realiza trote con desplazamientos anteroposteriores y laterales, con balón o sin balón (conducción). En esta fase, en las actividades de soporte y transferencia de peso en miembros inferiores pueden sumarse tareas de movimiento que involucren los miembros superiores, con lo cual se aumenta el grado de dificultad del ejercicio y, por ende, el beneficio será mayor.

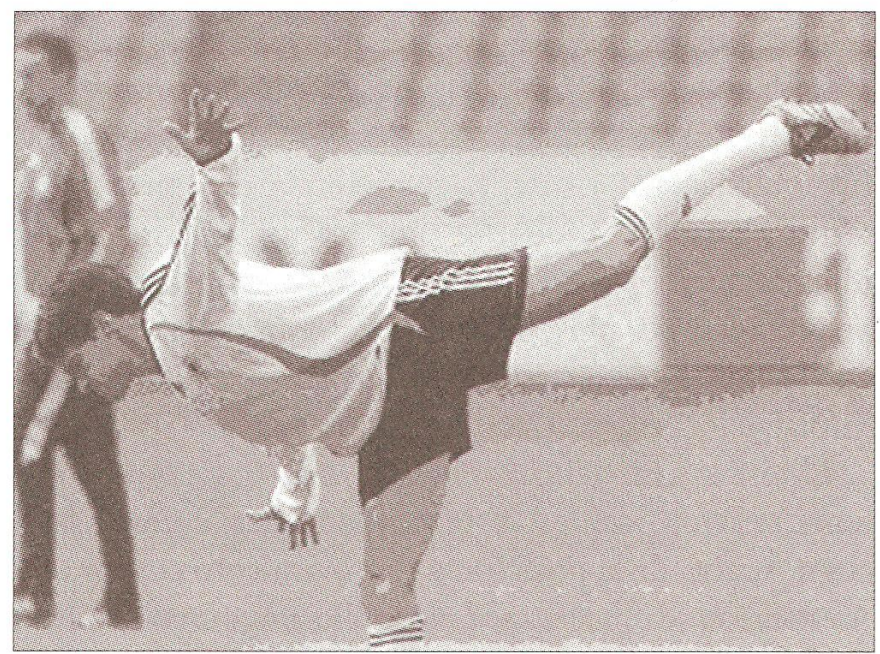

Tomado de: www.fifa.com (2006).

\section{Etapa 2}

Esta etapa es más dinámica, y complementando las tareas de transferencia y soporte de peso, se añaden actividades que impliquen desplazamientos en diferentes direcciones, con cambios de dirección (movimientos intempestivos, virajes, fintas), aceleraciones desde quietud y movimiento y desaceleraciones. Se realizan estas tareas simulando movimientos propios de la acción de juego, de acuerdo con la posición en la cual se desempeña el futbolista en el campo.

Para ello puede hacerse uso de diferentes elementos, como conos, aros, lazos, balones medicinales, pantalones de carga, paracaídas, para las actividades de desplazamiento en diferentes direcciones y ejercicios de movimientos combinados (posteriormente coordinación). Además, comienzan a generarse combinaciones de los patrones de movimiento específicos del fútbol: caminar, correr, saltar, patear, lanzar y atrapar con el pie, recibir y controlar el balón.

Así mismo, es importante evolucionar en el ascenso y descenso de rampas y escaleras con aumento de la velocidad de desplazamiento; y continuar haciendo uso de superficies como la arena, el prado, espuma, e incorporando elementos de la fundamentación técnica, como recepción del balón con diferentes partes del cuerpo, conducción, pase y remate, todas a modo de dominio del gesto técnico y no con las características específicas de la competencia. Por último, se inician trabajos pliométricos con la modalidad de multisaltos y, de acuerdo con la capacidad del deportista, se inician trabajos pliométricos con obstáculos a una altura de 15 a $30 \mathrm{~cm}$.

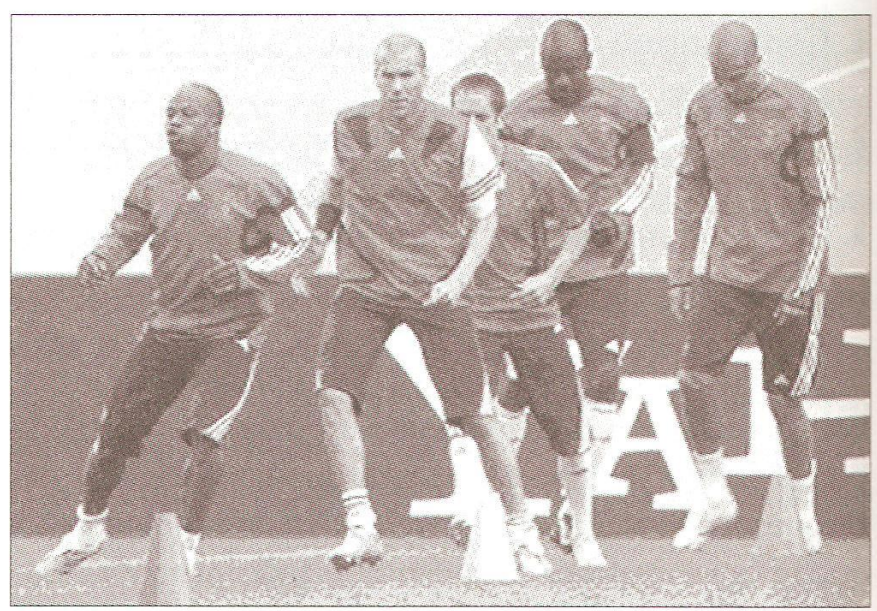

Tomado de: www.fifa.com (2006).

\section{Etapa 3}

Esta etapa hace énfasis en el desarrollo de actividades específicas del fútbol teniendo en cuenta si está desarrollándose un proceso preventivo o de rehabilitación, en el cual estarían ejecutándose tareas denominadas de retorno a la práctica deportiva.

Las tareas de movimiento deberán ser de mayor intensidad y a la vez mayor velocidad en los desplazamientos y los cambios de dirección (trabajo de espejo, acciones ofensivas y defensivas), en las aceleraciones y las desaceleraciones, en el manejo del centro de gravedad de acuerdo con la necesidad técnica.

Los patrones de movimiento deben ejecutarse en acciones de juego con uno o varios compañeros, con énfasis en situaciones momentáneas o variables, brevedad en el tiempo de preparación de la respuesta motriz, sin posibilidad de elección del momento de respuesta, inicio de respuesta desde posiciones inadecuadas para dicha respuesta, ajustes de movimiento de acuerdo con las 
necesidades, intervención de diversos factores externos que dificultan la tarea.

Se enfatiza en la técnica deportiva con tareas específicas de remate, centros, pase, regate, movimientos específicos de acciones defensivas y ofensivas; las cuales podrán ser ejecutadas con balón o sin balón, con adversario o sin él.

La velocidad en esta etapa debe ser casi normal, el entrenamiento pliométrico aumenta de acuerdo con las capacidades del futbolista.

\section{Etapa 4}

En esta etapa el futbolista deberá realizar actividades funcionales específicas del fútbol de un modo controlado, la velocidad y la intensidad de las tareas estarán determinadas por la capacidad motriz y física del atleta, su estabilidad funcional, su confianza; además, deberá insistirse sobre la integración de ejercicios específicos y la programación de actividades de rendimiento.

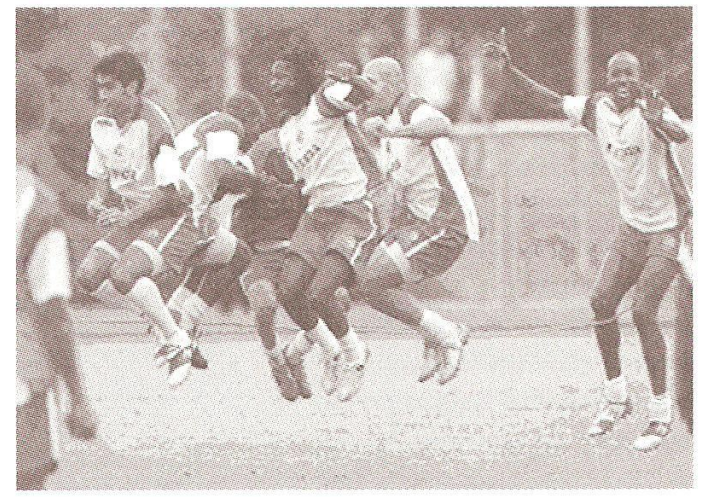

Tomado de: www.fifa.com (2006).

En esta etapa se perfecciona el programa de entrenamiento propioceptivo, por lo cual se requiere el análisis apropiado de las necesidades de rendimiento del futbolista y tener en cuenta las características específicas de sus funciones en el campo de juego (Laskowski; Lephart, 1998 y Swanik, 1997).

\section{Referencias}

Álvarez del Villar, C. (1992). La preparación física del fútbol basada en el atletismo. 3a. ed., Madrid: Editorial Gymnos, pp. 93-100.

Andrews, J. Physical rehabilitation of the injured athlete, 2a. ed. Alabama: Mosby, pp. 139-156.

Bosco, C. Aspectos fisiológicos de la preparación física del futbolista. 2a. ed. Barcelona: Paidotribo.

\section{Conclusiones}

Debe hacerse énfasis en que la reeducación propioceptiva es fundamental en todo proceso de entrenamiento preventivo o asistencial no sólo en el futbolista, también en las demás disciplinas deportivas. Con ésta se disminuye el riesgo de lesión y su reincidencia posterior a la rehabilitación. Deben tenerse en cuenta las características individuales, el tipo de deporte o ejercicio practicado, el nivel de entrenamiento y, sobre todo, la progresión del mismo, para facilitar así fenómenos de adaptación a estímulos externos, de manera que las respuestas de estabilización dinámica sean eficientes. Por consiguiente, el fisioterapeuta tiene una gran responsabilidad frente al mantenimiento o el mejoramiento de la reeducación propioceptiva.

El fisioterapeuta deberá concebir y ejecutar actividades creativas y técnicas que dinamicen el proceso de entrenamiento propioceptivo, teniendo además en cuenta que la lúdica representa un elemento importante en este proceso y que la eficacia del programa propioceptivo específico del deporte depende de que el fisioterapeuta conozca y entienda las necesidades propias del deporte, del deportista y la patología del atleta.

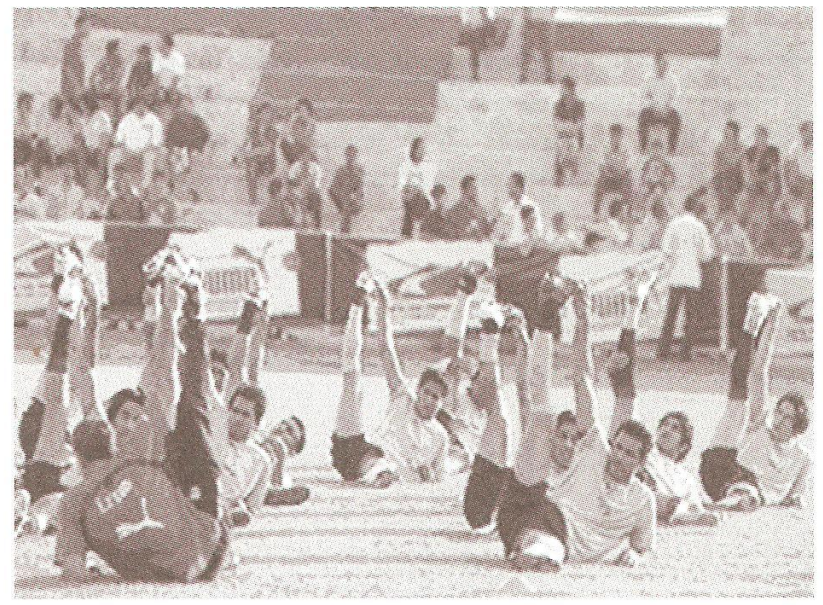

Tomado de: www.fifa.com (2006).

Csanadi, A. (1984). El fútbol. 3a. edición. Barcelona: Planeta, pp. 23-28.

Downie, P. (1993).Neurología para fisioterapeutas. 2a. ed. Buenos Aires: Editorial Médica Panamericana.

Gould, J. (1990). Orthopaedic and sports physical therapy. 2a. ed. St. Louis: Saunders, pp. 49-61. 
Guyton, A. (1991).Tratado de Fisiología Médica. 8a. ed. Madrid: McGraw-Hill, pp. 497-514, 529-540.

Kandel, E. y Schwartzs, J. (1991). Principles of Neural Science. 3a. ed. Connecticut: Editorial Appleton y Lange.

Laskowski, E. R. Rehabilitación perfeccionada con entrenamiento propioceptivo. The Physician and Sport Medicine.

Lephart, S. M. (1998). Propioception of the ankle and knee. Sports Medicine, 25 (3), 149-155.

Meinel, K. Didáctica del movimiento. La Habana: Pueblo y Educación, pp. 115-204.
Mount Castle, V. S. (1980). Medical Physiology. 14a. ed. St. Luis: Mosby.

Prentice, W, Técnicas de Rhb en la Medicina Deportiva. Barcelona: Paidotribo, pp. 138-157.

Sherrington, C. S. (1906). On the propioceptive system, especially in its reflex aspects. Brain, 29, 467-482.

Swanik, C. B. et al. (1997). Reestablishing propioception and neuromuscular control in the ACL-Injured Athlete. Journal of Sport Rehabilitation. pp. 6, 182, 206.

Zintl, Fritz. (1991). Entrenamiento de la resistencia. Barcelona: Ediciones Martínez Roca, pp. 15-30. 